\title{
Size matters
}

\section{Workshop on Growth Control in Development and Disease}

\section{Duojia Pan}

University of Texas Southwestern M edical Center at Dallas, D allas, Texas, USA

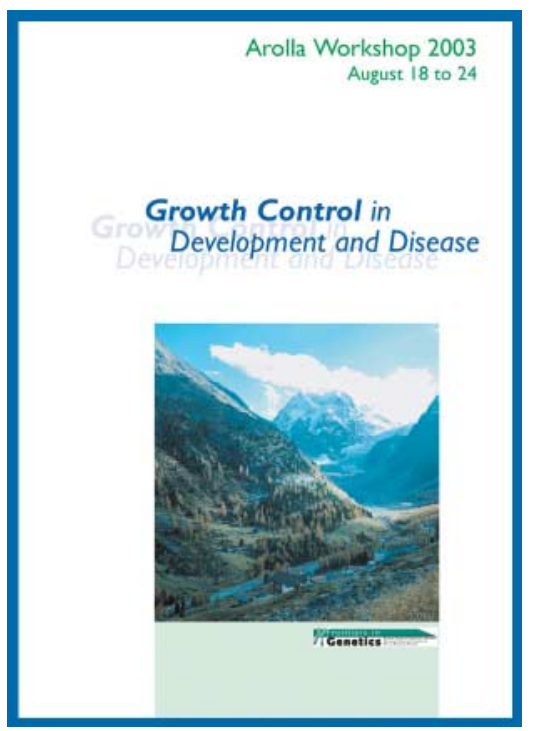

This Arolla workshop took place between 18 and 24 August 2003, and was organized by E. Hafen, D. Duboule and C. Kenyon

Keywords: cell competition; FOXO; insulin; longevity; sirtuins; TOR

EM BO reports (2004) 5, 136-139. doi:10.1038/s..embor.7400081

\section{Introduction}

A recent workshop entitled Growth Control in Development and Disease brought together around 90 scientists in the beautiful village of Arolla in the Swiss Alps. D uring this meeting, growth control was discussed from the perspectives of evolution, metabolism, ageing and cancer biology. From formal presentations to poster sessions, and mountain hikes to late-night discussions, the participants indulged in multidisciplinary scientific exchanges that stimulated innovative insights and forged new collaborations. Here, I present a few highlights from the meeting.

Department of Physiology, University of Texas Southwestern M edical Center at Dallas, $5323 \mathrm{H}$ arry Hines Boulevard, Dallas, Texas 75390-9040, USA

Tel: +1 214648 8487; Fax: +1 214648 8885;

E-mail: duojia.pan@utsouthwestern.edu

Submitted 17 November 2003; accepted 9 D ecember 2003; published online 23 January 2004
Systemic control of growth by hormones and growth factors Whereas the growth of single-cell organisms, such as yeast, is mainly regulated by nutrient availability, the growth of higher eukaryotes is dependent on the integration of many cell-extrinsic cues, which include hormones, growth factors and nutrients (Kimball \& Jefferson, 2000; Rohde et al, 2001). Indeed, the systemic control of metazoan grow th by hormones and nutrients is a topic of intense investigation in the growth-control field.

F. Nijhout (Durham, NC, USA) discussed how the final body size of insects is determined by the size at which a larva stops feeding and switches on the pupal fate (Nijhout, 2003). This transition from larval to pupal development requires the decay of juvenile hormone $(\mathrm{JH})$ and secretion of the moulting hormone ecdysone, the production of which, in turn, is regulated by the neurosecretory prothoracicotropic hormone (PTTH). Interestingly, different insects use diverse strategies to control the production of PTTH. In the tobacco hornworm M anduca sexta, the secretion of PTTH is induced by the cessation of JH, which, in turn, is tightly associated with the attainment of a well-defined 'critical weight'. In certain species of Hemiptera, such as the milkweed bug O ncopeltus fasciatus, PTTH secretion is controlled by abdominal stretch receptors that are activated when the animal reaches a particular size. By contrast, in the beetle $\mathrm{O}$ nthophagus taurus, PTTH secretion is triggered by the removal of its food supply, which in nature occurs when a larva exhausts the ball of food that is provided by its mother. Although a lot of research is still required to understand the molecular mechanisms that underpin these phenomena, it is reasonable to conclude that knowledge of both neural physiology and behaviour is necessary to understand size control at the organismal level.

Much progress has been made in recent years towards understanding the role of insulin-like molecules in promoting cell growth. Insulin-like molecules are present in worms, insects and mammals, and are part of an evolutionarily conserved signalling pathway that involves the insulin receptor (INR), insulin-receptor substrate (IRS), phosphatidylinositol-3-O H kinase (PI(3)K), phosphoinositide-dependent kinase 1 (PDK1) and the protein kinase AKT (Fig 1). Several presentations focused on the molecular dissection of this pathway. S. Leevers (London, UK) discussed a genetic screen to identify new components of the insulin pathway in Drosophila. From this screen, she isolated a gene, pixie, which 


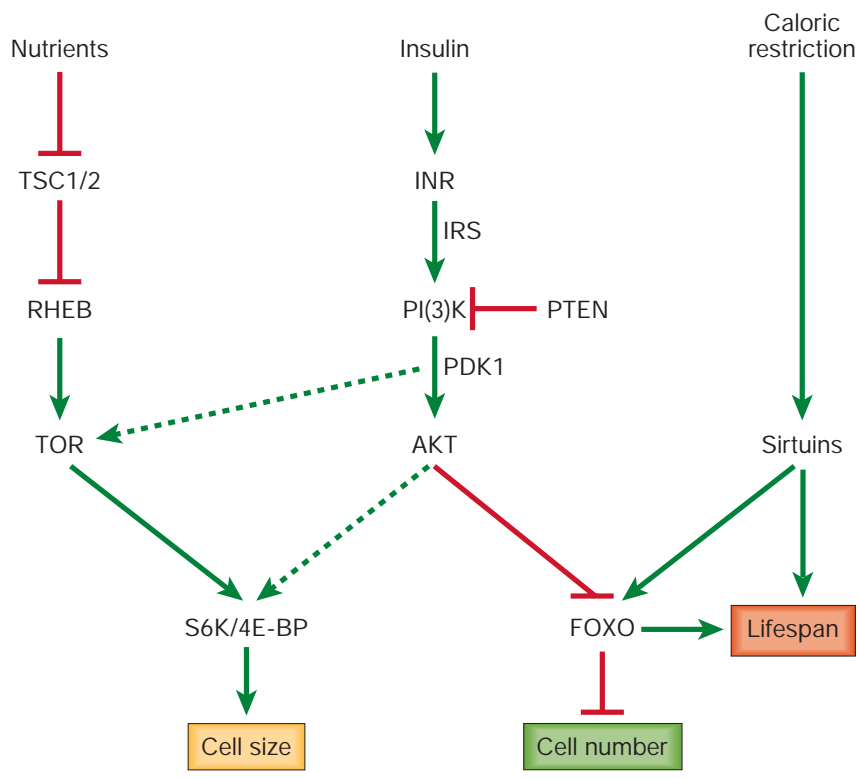

Fig1| Cross talk between growth control and ageing pathways. Depicted are theinsulin-signalling pathway, the nutrient-sensing TSC/TOR pathway and thecaloric restriction-responsivesirtuins pathway. FOXO, forkhead transcription factor; IN R, insulin receptor; IRS, insulin-receptor substrate; PDK 1, phosphoinositide-dependent kinase 1; PI(3)K, phosphatidylinositol3-OH kinase; PTEN, phosphatase and tensin homologue; RHEB, RAS homologue enriched in brain; TOR, target of rapamycin; TSC, tuberoussclerosis complex.

encodes a soluble ATP-binding cassette $(A B C)$ domain protein. Data were presented that implicate Pixie in the general control of protein translation. E. Hafen (Zürich, Switzerland) discussed studies of the Drosophila homologue of the forkhead transcription factor FOXO, which in Caenorhabditis elegans has been shown to act downstream of AKT in the ageing pathway. FOXO seems to specifically mediate the reduction in cell numbers in insulin-signalling mutants through the transcriptional regulation of target genes, including the translation-initiation regulator 4E-BP (Jünger et al, 2003; Fig 1). B. Burgering (U trecht, the $N$ etherlands) and A. Brunet (Boston, MA, USA) discussed how the FOXO family function as central molecular sensors of the extracellular environment in mammalian cells (Burgering \& Kops, 2002; Tran et al, 2003). These studies indicate that different patterns of phosphorylation and acetylation of $\mathrm{FOXO}$ proteins might specify various cellular responses that range from stress resistance to apoptosis. The coregulation of FOXO proteins by AKT and protein deacetylases, such as sirtuins (see below), provides an important link between the two main longevity pathways of insulin signalling and caloric restriction (Fig 1). M. White (Boston, MA, USA) discussed the insights that have been obtained from studying mice in which Irs2 is specifically disrupted in the pancreatic $\beta$-cells and the hypothalamus. This mouse model recapitulates many of the classic features of type 2 diabetes, including the development of diabetes and obesity. Restoring Irs2 expression in the $\beta$-cells rescues the diabetic phenotype without reversing the obesity phenotype. Therefore, Irs2 signalling in the hypothalamus seems to be important for the regulation of food intake.

\section{Regulation of growth by nutrients}

It has long been appreciated that nutrient availability is a crucial determinant of body size, but the molecular mechanisms that underlie this phenomenon are only just beginning to be elucidated. Several insulin-like peptides are expressed in Drosophila neurosecretory cells in the larval brain in a nutrient-dependent manner, which indicates that nutrients might promote growth through the regulation of insulin production (Ikeya et al, 2002). The fat body of Drosophila, which is an endocrine organ that is analogous to the mammalian liver, also produces a systemic signal that regulates larval growth in response to nutrients (Britton \& Edgar, 1998). $\mathrm{P}$. Leopold (N ice, France) presented molecular evidence for this fatbody-derived systemic signal (Colombani et al, 2003). The specific downregulation of amino-acid uptake in the fat body, through the inhibition of an amino-acid transporter called slimfast, causes a global growth defect similar to that seen in Drosophila that are raised under poor nutrient conditions. In the fat-body cells, slimfast regulates the production of a secreted cofactor of insulin-like molecules through a pathway that involves the tuberous-sclerosis complex tumour-suppressor proteins ( $\mathrm{Tsc} 1 / \mathrm{Tsc} 2$ ) and target of rapamycin (Tor) (for details of the general TSC/TOR pathway, see Fig 1). This cofactor, which is known as the acid-labile subunit (Als), then acts systemically to control the growth of peripheral tissues through the local regulation of insulin signalling. M. Galloni (Montpellier, France) presented evidence that the larval gut is also involved in the systemic control of growth in Drosophila. Mutations of bonsaï, which is predominantly expressed in the gut, result in the retardation of systemic growth. Therefore, the ability to mediate the systemic control of organismal growth is probably not limited to the neurosecretory cells of the brain and the fat body.

Besides their systemic mode of action, nutrients also exert profound cell-autonomous effects on cell growth. The TOR-signalling pathway has emerged as a central pathway that couples nutrient availability to cellular growth. M. Hall (Basel, Switzerland) and D. Sabatini (Boston, MA, USA) discussed recent progress in the characterization of theTO R-signalling complex (TO RC) in yeast (Loewith et al, 2002) and mammalian cells (Kim et al, 2002), respectively. TOR exists in two distinct functional complexes, with TO RC1 mediating the rapamycin-sensitive pathway and TORC2 mediating the rapamycin-insensitive pathway. Hall also presented data indicating that TO RC1 might control ribosome biogenesis through the RAS and cyclic AMP pathway in yeast. T. Radimerski (Basel, Switzerland) and D. Pan (Dallas, TX, USA) presented recent studies in D rosophila that have implicated Tsc1/Tsc2 and the small GTPase Ras homologue enriched in brain (Rheb) as upstream regulators of Tor (Garami et al, 2003; Saucedo et al, 2003; Stocker et al, 2003; Zhang et al, 2003). Collectively, these studies show that the TSC1/TSC 2 protein complex functions as a GTPase activating protein (GAP) of RHEB, which, in turn, regulatesTO R activity. Pan al so presented evidence that during Drosophila development, the Tsc/Rheb pathway acts in parallel to, rather than downstream of, the insulin/PI(3)K/Akt pathway.

\section{Insulin signalling, caloric restriction and ageing}

The insulin-signalling pathway not only controls the growth of an organism, but also exerts a profound influence on metabolism, reproduction and longevity. Several talks focused on the molecular and physiological aspects of ageing. In C. elegans, a decrease in signalling through the insulin receptor DAF-2 results in a DAF-16 (a FOXO orthologue)-dependent increase in longevity. C. Kenyon (San 
Francisco, CA, USA) and G. Ruvkun (Boston, MA, USA) discussed recent efforts to identify the transcriptional targets of DAF-16 in C. elegans (Lee et al, 2003; M urphy et al, 2003). Collectively, these studies show that DAF-16 functions both as a transcriptional activator and a transcriptional repressor. Downstream targets of DAF-16 include genes that are implicated in diverse cellular processes, such as metabolism, stress response and antimicrobial defence. It seems that individual targets have a relatively minor effect on lifespan and that all DAF-16 targets act additively to extend longevity.

Caloric restriction slows the pace of ageing and increases the maximum lifespan of species as diverse as yeast, worm, flies and mammals. Two presentations at the meeting provided exciting insights into this phenomenon. L. Partridge (London, UK) reported that in Drosophila, dietary restriction (DR) prolongs lifespan by reducing the short-term risk of death ( $M$ air et al, 2003). She noted that DR in adult flies of any age generates equal protection from mortality, irrespective of previous dietary history. These results indicate that caloric restriction does not increase longevity by simply reducing the accumulation of cellular damage. However, although it could be inferred from these studies that there is no urgent need to start good eating habits early in life, Partridge cautioned that comparable experiments have yet to be performed in mammals. D. Sinclair (Boston, MA, USA) discussed the discovery of small molecules that mimic the effects of caloric restriction (H owitz et al, 2003). These molecules, including resveratrol-which is a plant polyphenol that is commonly found in red wine-stimulate the enzymatic activity of a family of NAD+-dependent protein deacetylases called sirtuins, which, in turn, extend the lifespan of yeast and mammalian cells. Therefore, sirtuin activators might be exploited for the therapeutic intervention of ageing-related human diseases.

\section{Regulation of growth at the tissue level}

Studies of the insulin and the TSC/TOR pathways indicate that environmental cues, such as nutrients, are important in determining the final size of an organ. However, there must be intrinsic counting mechanisms that stop growth when an organ reaches its final size. This phenomenon was the subject of many intense discussions at the meeting and how an organ (or organism) stops growing remains an open question.

D. Ingber (Boston, MA, USA) discussed how mechanical forces might be transduced into alterations in cellular biochemistry. $\mathrm{He}$ noted that extracellular matrix (ECM)-dependent changes in cell shape affect the decision of a cell to proliferate, differentiate, die or migrate (Ingber, 2002). His presentation reinforced the importance of considering size control at the tissue level and reminded us of the important contribution of the ECM, which is unarguably a little-studied aspect in the grow th-control field.

During the growth of $D$ rosophila imaginal discs, cells compete for limited survival factors. As a consequence, when slow-growing cells are juxtaposed with those that are fast growing, the slow-growing cells are actively eliminated by cell death-a phenomenon that is known as cell competition. L. Johnston (New York, NY, USA) and K. Basler (Zürich, Switzerland) offered molecular insights into this process. They reported that the myc oncogene has a key role in cell competition. Elevated M yc levels are sufficient to transform cells into 'super competitors'. Conversely, the manipulation of several other growth pathways, such as insulin/PI(3)K and cyclin D, does not result in altered cell competition. This differential effect is probably owing to the fact that the downstream targets that are activated by Myc are different to those that are activated by insulin/PI(3)K. B. Edgar (Seattle, WA, USA) has investigated these downstream targets and his studies show that M yc preferentially stimulates ribosome biogenesis.

Although cell competition represents an important mechanism for regulating local cell-cell interactions, this does not seem to account for the 'mass checkpoint' that stops growth when an organ reaches its final size. Drosophila genetics might provide a powerful tool to probe the molecular mechanism of this elusive checkpoint. G. Halder (Houston, TX, USA), I. Hariharan (Boston, MA, USA) and Pan reported recent findings that implicate the tumour-suppressor genes hippo, salvador and warts in this process (H arvey et al, 2003; Udan et al, 2003; Wu et al, 2003). The inactivation of any of these genes results in the increased transcription of the cell-cycle regulator cyclin E and the cell-death inhibitor diap1, which leads to increased proliferation and reduced apoptosis. These tumour suppressors define a new kinase cascade in which Hippo phosphorylates Salvador and Warts, and Salvador potentiates the kinase activity of Hippo towards Warts. By analogy to other kinase cascades, it is not unreasonable to speculate that this pathway might be triggered by an unknown ligand. It can be envisioned that this ligand might be present at low concentrations during the exponential growth of imaginal discs and that its concentration increases as the disc approaches its final size. When it reaches this point, the Hippo pathway is fully activated, and growth is arrested by the triggering of cell death and the inhibition of cell proliferation. Another example of the coordination of cell proliferation and death was provided by N. Baker (N ew York, NY, USA), who showed that Egf-receptor signalling mediates both processes in the D rosophila eye (Baker \& Yu, 2001).

\section{G rowth and cancer}

Cancer occurs when grow th control goes awry. J. Lees (Boston, MA, USA) described a genetic screen for tumour-suppressor genes in zebrafish that is being conducted in collaboration with $\mathrm{N}$. Hopkins (Boston, MA, USA). A surprising finding from the screen is that heterozygous mutations for many ribosomal proteins predispose animals to tumour formation. The underlying mechanisms for the involvement of ribosomal proteins in tumorigenesis are unknown. T. Tlsty (San Francisco, CA, USA) discussed a cancer-progression model that uses human mammary epithelial cells (HMECs) (Romanov et al, 2001). She described the stereotypical molecular changes in this cell-culture model, including the inactivation of p16 and the failure to maintain genomic instability. She also discussed how such changes mimic many of those seen in premalignant lesions in breast cancer, and how observations of this model could identify potential targets for prevention and therapy.

A growing trend in cancer therapy is to develop drugs that specifically target the oncogenic or tumour-suppressor pathway that is dysregulated in a particular tumour. This approach is based on the concept that tumours often become 'addicted' to certain dysregulated signalling pathways and hence become hypersensitive (compared with normal cells) to their downregulation (Weinstein, 2002). Therefore, anticancer drugs that target such dysregulated pathways present the best opportunity to achieve high efficacy at low toxicity. A. M atter (Basel, Switzerland) spoke about the history of the development of G leevac, which is a small-molecule inhibitor of the oncogenic tyrosine kinase BCR-ABL for the treatment of chronic myelogenous leukaemia (CML). The potency of Gleevac against other tyrosine kinases, including c-KIT and the platelet-derived growth factor (PDGF) receptor, is also exploited for the treatment of several other diseases. 


\section{Concluding remarks}

Impressive progress has been made in recent years in elucidating certain molecular aspects of growth control in metazoan development. How ever, our knowledge remains rudimentary. At the cellular level, components of many growth-regulating pathways have yet to be identified. At the tissue level, the mass checkpoint that stops growth when an organ reaches its final size remains elusive. At the organismal level, we are far from understanding the regulatory interplay among the tissues that modulate growth in intact animals. The multidisciplinary approach of this meeting certainly reminded the participants to think about the 'bigger picture' of growth control from a range of perspectives, including evolution, ecology, physiology, genetics and cell biology. W ith the integration of several approaches, the field of growth control is destined to progress rapidly in the coming years. Hopefully, a future reunion in the scenic Arolla village will show how much the field has accomplished.

\section{ACKN O W LED GEMENTS}

Financial support for the Arolla workshop 2003 was kindly provided by the National Center of Competence in Research (NCCR) Frontiers in Genetics, Kontaktgruppe für Forschungsfragen (KGF), J. Klaus-Stiftung, G. and A. Claraz-Schenkung, Novartis Pharma AG, Schweizerischer Nationalfonds, Serono International S.A., Zürcher Universitätsverein, Krebsliga Schweiz, Jubiläumsstiftung der Zürich Versicherungs-Gruppe, SchweizerischeA kademie der M edizinischen Wissenschaften, Applied Biosystems, M icrosynth GmbH , Rahn \& Bodmer Banquiers, Leica M icrosystems, SchweizerischeA kademie der Naturwissenschaften and The Genetics Company. Excellent administrative support was provided by K. Widmer (Zürich, Switzerland).

\section{REFERENCES}

Baker NE, Yu SY (2001) The EG F receptor defines domains of cell cycle progression and survival to regulate cell number in the developing Drosophila eye. Cell 104: 699-708

Britton JS, Edgar BA (1998) Environmental control of the cell cycle in D rosophila: nutrition activates mitotic and endoreplicative cells by distinct mechanisms. Development 125: 2149-2158

Burgering BM , Kops GJ (2002) Cell cycle and death control: long live forkheads. Trends Biochem Sci 27: 352-360

Colombani J, Raisin S, Pantalacci S, Radimerski T, M ontagne J, Leopold P (2003) A nutrient sensor mechanism controls D rosophila growth. Cell 114: 739-749

Garami A, Zwartkruis FJ, N obukuni T, Joaquin M, Roccio M, Stocker H, Kozma SC, Hafen E, Bos JL, Thomas G (2003) Insulin activation of Rheb, a mediator of $\mathrm{mTO}$ R/S6K/4E-BP signaling, is inhibited by TSC1 and 2. Mol Cell 11: 1457-1466

Harvey KF, Pfleger CM, H ariharan IK (2003) The D rosophila M st ortholog, hippo, restricts growth and cell proliferation and promotes apoptosis. Cell 114: 457-467

Howitz KT et al (2003) Small molecule activators of sirtuins extend Saccharomyces cerevisiae lifespan. $N$ ature 425: 191-196

Ikeya T, Galic M, Belawat P, Nairz K, H afen E (2002) Nutrient-dependent expression of insulin-like peptides from neuroendocrine cells in the CNS contributes to growth regulation in D rosophila. Curr Biol 12: 1293-1300

Ingber DE (2002) Mechanical signaling and the cellular response to extracellular matrix in angiogenesis and cardiovascular physiology. Circ Res 91: 877-887
Jünger MA, Rintelen F, Stocker H, Wasserman JD, Vegh M, Radimerski T, Greenberg ME, H afen E (2003) The D rosophila forkhead transcription factor FOXO mediates the reduction in cell number associated with reduced insulin signaling. J Biol 2: 20

Kim DH, Sarbassov DD, Ali SM, King JE, Latek RR, Erdjument-Bromage $H$, Tempst P, Sabatini D M (2002) mTO R interacts with raptor to form a nutrient-sensitive complex that signals to the cell growth machinery. Cell 110: 163-175

Kimball SR, Jefferson LS (2000) in Translational Control of Gene Expression (eds Sonenberg JW, Hershey JW B, M athews M B), 561-579. Cold Spring Harbor Laboratory Press, Cold Spring H arbor, N ew York, USA

Lee SS, Kennedy S, Tolonen AC, Ruvkun G (2003) DAF-16 target genes that control C. elegans life-span and metabolism. Science 300: 644-647

Loewith R, Jacinto E, W ullschleger S, Lorberg A, Crespo JL, Bonenfant D, O ppliger W, Jenoe P, Hall M N (2002)Two TO R complexes, only one of which is rapamycin sensitive, have distinct roles in cell growth control. Mol Cell 10: 457-468

M air W, G oymer P, Pletcher SD, Partridge L (2003) Demography of dietary restriction and death in D rosophila. Science 301: 1731-1733

Murphy CT, M cCarroll SA, Bargmann CI, Fraser A, Kamath RS, Ahringer J, $\mathrm{Li} \mathrm{H}$, Kenyon C (2003) Genes that act downstream of DAF-16 to influence the lifespan of Caenorhabditis elegans. Nature 424: 277-283

Nijhout HF (2003) The control of body size in insects. Dev Biol 261: 1-9

Rohde J, Heitman J, Cardenas M E (2001) The TO R kinases link nutrient sensing to cell growth. J Biol Chem 276: 9583-9586

Romanov SR, Kozakiewicz BK, Holst CR, Stampfer M R, Haupt LM, Tlsty TD (2001) N ormal human mammary epithelial cells spontaneously escape senescence and acquire genomic changes. N ature 409: 633-637

Saucedo LJ, Gao X, Chiarelli DA, Li L, Pan D, Edgar BA (2003) Rheb promotes cell growth as a component of the insulin/TO R signalling network. $\mathrm{N}$ at Cell Biol 5: 566-571

Stocker H, Radimerski T, Schindelholz B, Wittwer F, Belawat P, Daram P, Breuer S, Thomas G, H afen E (2003) Rheb is an essential regulator of S6K in controlling cell growth in Drosophila. Nat Cell Biol 5: 559-566

Tran H, Brunet A, G riffith EC, G reenberg ME (2003)The many forks in FO XO's road. SCI STKE 2003: RE5

U dan RS, Kango-Singh M, N olo R, Tao C, Halder G (2003) Hippo promotes proliferation arrest and apoptosis in the Salvador/W arts pathway. N at Cell Biol 5: 914-920

Weinstein IB (2002) Addiction to oncogenes- the Achilles heal of cancer. Science 297: 63-64

Wu S, Huang J, D ong J, Pan D (2003) hippo encodes a Ste-20 family protein kinase that restricts cell proliferation and promotes apoptosis in conjunction with salvador and warts. Cell 114: 445-456

Zhang Y, Gao X, Saucedo LJ, Ru B, Edgar BA, Pan D (2003) Rheb is a direct target of the tuberous sclerosis tumour suppressor proteins. N at Cell Biol 5: $578-581$

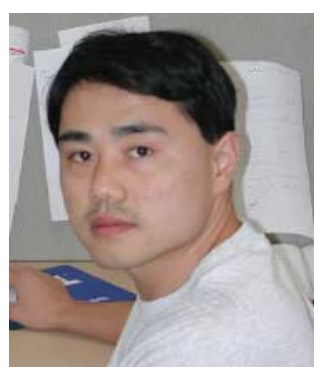

Duojia Pan 